\title{
DOA estimation algorithm for high maneuvering scenarios Based on improved covariance matrix
}

\author{
Zhengtang Liu,Yanjie Cheng,Hui Ma,Wen Yang ,Huimin Gao\&Dongdong Zhou \\ LuoYang Electronic Equipment Test Center, Luoyang, Henan,471000,China \\ E-mail:18638806677@126.com
}

Keywords: Array signal processing; Coherent signal; DOA; Covariance matrix.

\begin{abstract}
Aiming at the problem that signal snapshot data of the target is sparse and conventional algorithm is difficult to obtain the signal subspace ,a new DOA decorrelation algorithm based on improved covariance matrix is proposed. Firstly, the received single snapshot data is pretreated by cross-correlation, then the equivalent covariance matrix is reconstructed by using the data obtained from the pretreatment, and then the DOA estimation of coherent signals is completed based on the MUSIC algorithm. Without losing the aperture of the array ,the proposed algorithm ensures the accuracy of the spectral estimation . Simulation results demonstrate that the proposed algorithm can achieve better performance than that of traditional method in high-speed mobile scenarios.
\end{abstract}

\section{Introduction}

Because of its high detection accuracy and fast estimation speed, Signal detection and estimation based on array antenna has been widely used in many fields such as radar, sonar, radio communication, navigation and many other fields ${ }^{[1]}$. When the DOA estimation of target signal is carried out on the missile borne, airborne and other high speed moving platform, the real-time performance requirement is high, and the environment of the array antenna is complex and changeable. The received valid data is generally a few single snapshot data under strong interference environment. In this case traditional methods such as spatial smoothing algorithm ${ }^{[2]}$,the vector singular value decomposition algorithm ${ }^{[3]}$, the Toeplitz matrix reconstruction algorithm ${ }^{[4]}$, and so on can not be able to obtain approximate signal subspace, and can not be able to get the target signal's DOA information. Therefore, it is a solution to estimate the $\mathrm{DOA}^{[5-8]}$ of coherent signals using single snapshot data.

This paper proposes a DOA estimation algorithm of coherent signals based on equivalent covariance matrix. The single snapshot data of all array are pretreatment, using income data, the equivalent covariance matrix is reconstructed, then the DOA estimation is carried out by using the music algorithm. Theoretical analysis and computer simulation demonstrate that without lose the array aperture, the proposed algorithm in this paper requires a smaller amount of snapshot, and fewer computation, which is suitable for DOA estimation in high-speed mobile scenarios.

\section{Signal Model}

Assuming that the receiving antenna array is m-element isotropic uniform linear array .The array element spacing $d=\lambda / 2$.In the observation period, a total of $N$ far field narrowband coherent signal is received. The incident angle are respectively: $\sigma_{i}^{2} 、 \theta_{i}, i=1, \ldots, J, \ldots, N$. With first elements as the reference array, the received signal of the array element $m$ at the time can be expressed as:

$$
x_{m}(t)=\sum_{i=1}^{N} s_{i}(t) \exp \left\{j(m-1) \pi \sin \left(\theta_{i}\right)\right\}+n_{m}(t)
$$

In the formula, $s_{i}(t)$ is the complex envelope of the signal $i ; n_{m}(t)$ is zero mean AWGN of array

$m$ at time $t$, These noises are not correlated in space and time field, with power of $\sigma_{n}^{2}$.

The formula (1) can be written as a vector: 


$$
\boldsymbol{X}(t)=\boldsymbol{A}(\boldsymbol{\theta}) \boldsymbol{S}(t)+\boldsymbol{N}(t)
$$

Among the formula, $\boldsymbol{A}(\boldsymbol{\theta})$ is the $M \times N$ order array manifold matrix; $\boldsymbol{S}(t)$ is the input signal vector, and $N(t)$ is the noise vector.

Then the data covariance matrix of array signal can be written as:

$$
\boldsymbol{R}=E\left\{\boldsymbol{X} \boldsymbol{X}^{\mathrm{H}}\right\}=\sum_{k=1}^{\infty} \boldsymbol{X}(t) \boldsymbol{X}(t)^{\mathrm{H}}
$$

\section{Algorithm Description}

The basic idea of DOA estimation for coherent signal is to construct an equivalent covariance matrix whose rank is equal to the number of incident signal by using the snapshot data. The form of equivalent covariance matrix should be satisfied as:

$$
R=A(\theta) R_{s} A(\theta)^{\mathrm{H}}
$$

According to above ideas, the covariance matrix is firstly constructed as follows:

$$
\boldsymbol{R}_{1}=\left[\begin{array}{ccccc}
x_{1}(t) & x_{2}^{*}(t) & x_{3}^{*}(t) & \mathrm{L} & x_{N}^{*}(t) \\
x_{2}(t) & x_{1}(t) & x_{2}^{*}(t) & \mathrm{L} & x_{N-1}^{*}(t) \\
x_{3}(t) & x_{2}(t) & x_{1}(t) & \mathrm{L} & x_{N-2}^{*}(t) \\
\mathrm{M} & \mathrm{M} & \mathrm{M} & \mathrm{O} & \mathrm{M} \\
x_{N}(t) & x_{N-1}(t) & x_{N-2}(t) & \mathrm{L} & x_{1}(t)
\end{array}\right]
$$

In order to satisfy the formula (4), the snapshot data must satisfy the $s_{i}(t)=s_{i}^{*}(t)$, which requires a higher demand for the received signal. In order to deal with the received signals under different conditions, the equivalent covariance matrix $\boldsymbol{R}_{1}$ needs to be modified. With the snapshot data of the first element as reference, the cross correlation processing is carried out on the $\boldsymbol{R}_{1}$, and the new equivalent covariance matrix $\boldsymbol{R}_{2}$ is obtained. $\boldsymbol{R}_{2}$ satisfy the following relations

$$
\boldsymbol{R}_{2}(i, j)=\left\{\begin{array}{cc}
R_{1}(i, j) x_{1}(t) & i<j \\
R_{1}(i, j) x_{1}^{*}(t) & \text { others }
\end{array}\right.
$$

After the above processing, the equivalent covariance matrix $\boldsymbol{R}_{2}$ form is as follows:

$$
\boldsymbol{R}_{2}=\left[\begin{array}{ccccc}
x_{1}(t) x_{1}^{*}(t) & x_{2}^{*}(t) x_{1}(t) & x_{3}^{*}(t) x_{1}(t) & \mathrm{L} & x_{N}^{*}(t) x_{1}(t) \\
x_{2}(t) x_{1}^{*}(t) & x_{1}(t) x_{1}^{*}(t) & x_{2}^{*}(t) x_{1}(t) & \mathrm{L} & x_{N-1}^{*}(t) x_{1}(t) \\
x_{3}(t) x_{1}^{*}(t) & x_{2}(t) x_{1}^{*}(t) & x_{1}(t) x_{1}^{*}(t) & \mathrm{L} & x_{N-2}^{*}(t) x_{1}(t) \\
\mathrm{M} & \mathrm{M} & \mathrm{M} & \mathrm{O} & \mathrm{M} \\
x_{N}(t) x_{1}^{*}(t) & x_{N-1}(t) x_{1}^{*}(t) & x_{N-2}(t) x_{1}^{*}(t) & \mathrm{L} & x_{1}(t) x_{1}^{*}(t)
\end{array}\right]
$$

Through the pretreatment of the sample data of the reference array, the formula (7) contains the signal itself, which also contains the signal conjugated. From construction principle of the formula (7), it can be known that the elements in $\boldsymbol{R}_{2}$ meet the $\boldsymbol{R}_{2}(i, j)=\boldsymbol{R}_{2}^{*}(i, j)$, that is, the matrix $\boldsymbol{R}_{2}$ is the Hermite matrix. At the same time, it is equal to the elements in any a straight line which is parallel to the main diagonal. That is, the matrix $\boldsymbol{R}_{2}$ is the Toeplitz matrix, which satisfies the basic requirement of the reconstruction matrix. The following proves that the rank of $\boldsymbol{R}_{2}$ is equal to the number of incident signals, which form is satisfied with formula (4).

First look at any of the triangular elements $\boldsymbol{R}_{2}(i, j)$ in the matrix $\boldsymbol{R}_{2}$ with $i \geq j$ 


$$
\begin{aligned}
\boldsymbol{R}_{2}(i, j) & =x_{i}(t) x_{1}^{*}(t) \\
& =\left(\sum_{n=1}^{N} s_{n}(t) \exp \left\{j(i-1) \pi \sin \left(\theta_{n}\right)\right\}+n_{i}(t)\right)\left(\sum_{p=1}^{N} s_{p}^{*}(t)+n_{1}^{*}(t)\right) \\
& =\sum_{n=1}^{N} \sum_{p=1}^{N} s_{p}^{*}(t) s_{n}(t) \exp \left\{j(i-1) \pi \sin \left(\theta_{n}\right)\right\}+\sigma^{2} \delta(i) \\
& =\boldsymbol{a}_{i}(\boldsymbol{\theta}) \boldsymbol{R}_{s} \boldsymbol{a}_{1}^{H}+\sigma^{2} \delta(i)
\end{aligned}
$$

Among formula(8), $\boldsymbol{a}_{i}(\boldsymbol{\theta})$ is the $i$ th line of the array manifold matrix $\boldsymbol{A}(\boldsymbol{\theta})$,

$$
\begin{aligned}
& \boldsymbol{a}_{i}(\boldsymbol{\theta})= {\left[\exp \left\{j(i-1) \pi \sin \left(\theta_{1}\right)\right\}, \exp \left\{j(i-1) \pi \sin \left(\theta_{2}\right)\right\} \mathrm{L}\right.} \\
&\left.\exp \left\{j(i-1) \pi \sin \left(\theta_{M}\right)\right\}\right] \\
& \boldsymbol{R}_{s}=\operatorname{diag}\left(\sum_{n=1}^{N} s_{n}^{*}(t) s_{1}(t), \sum_{n=1}^{N} s_{n}^{*}(t) s_{2}(t) \mathrm{K} \sum_{n=1}^{N} s_{n}^{*}(t) s_{N}(t)\right)
\end{aligned}
$$

$\sigma^{2}$ is noise power, $\delta(i)=\left\{\begin{array}{cc}1, & \mathrm{i}=1 \\ 0, & \text { others }\end{array}\right.$. By the noise of the hypothesis, $\sigma^{2}$ is only a reference to the noise, and only on the diagonal of the matrix $\boldsymbol{R}_{2}$, the noise of other array after the pretreatment of the formula(6) has been effectively suppressed.

Similarly, any lower triangular element $\boldsymbol{R}_{2}(j, i), i \geq j$ of the matrix $\boldsymbol{R}_{2}$ can be expressed as:

$$
\boldsymbol{R}_{2}(j, i)==\boldsymbol{a}_{1}^{H} \boldsymbol{R}_{s} \boldsymbol{a}_{j}(\boldsymbol{\theta})
$$

According to the formula (9) and (11), it shows that the matrix $\boldsymbol{R}_{2}$ can be expressed as follows:

$$
\boldsymbol{R}_{2}=\boldsymbol{A}(\boldsymbol{\theta}) \boldsymbol{R}_{\mathrm{s}} \boldsymbol{A}(\boldsymbol{\theta})^{\mathrm{H}}+\sigma_{n}^{2} \boldsymbol{I}_{N \times N}
$$

In the formula(12), $\boldsymbol{A}(\boldsymbol{\theta})$ is the Vandermonde matrix, and the incidence angle $\theta_{i}$ is not the same, so the $\boldsymbol{A}(\boldsymbol{\theta})$ is the column full rank matrix, whose Rank is N; The rank of $\boldsymbol{R}_{2}$ is diagonal matrix, and the signal is not zero, so the rank of the reconstructed matrix is equal to the number of incident signals. The correct signal subspace and feature subspace can be obtained by eigen value decomposition of the matrix $\boldsymbol{R}_{2}$. Based on the reconstruction of the equivalent covariance matrix $\boldsymbol{R}_{2}$, the MUSIC algorithm is used to solve the incident signal DOA. So the following steps of the proposed algorithm are summarized in this paper:

Step1: get the single snapshot data of the array receiving; Step2: construct the equivalent covariance matrix $\boldsymbol{R}_{1}$; Step 3: using $\boldsymbol{R}_{1}$ and the data of reference array to preprocess the data of single snapshot; Step 4: construct the equivalent covariance matrix $\boldsymbol{R}_{2}$; Step 5: carry out DOA estimation by using MUSIC algorithm.

\section{Simulation Analysis}

In this section, we present several simulation examples to illustrate the performance of the proposed algorithm as compared to some of the existing methods. In order to verify the algorithm's effectiveness, the proposed algorithm (abbreviated as single snapshot ,SSP) and Toeplitz algorithm ,spatial smoothing algorithm (abbreviated as SSM)and singular value decomposition algorithms (abbreviated as SVD) are compared through the simulation test.

We assumed that the array is uniform linear array with 8 sensors and the antenna spacing is half wavelength. The additive noise is zero mean uniform white noise, and the target signals are coherent signals.

Simulation 1: the influence of snapshot numbers on the algorithm.

In this simulation, we assumed that three target signals of equal power with angles $\theta_{1}=-40^{\circ}, \theta_{2}=-20^{\circ}, \theta_{3}=0^{\circ}$ and $S N R=20 \mathrm{~dB}$, using music algorithm for spectral peak searching, the searching precision is $1^{\circ}$. When the snapshot number is 1000 , the simulation results of the algorithm 
of Toeplitz, SSM, SVD and SSP are shown in Figure.1. When the snapshot number is 1 with other simulation conditions not changed, the simulation results are shown in Figure 2. It can be concluded from Fig. 1 that when the snapshot number is large, all algorithm can accurately estimate coherent signal DOA, but the spectrum peak of proposed algorithm is sharper than the other three algorithms. It can be seen clearly from Fig.2 that when the snapshot number is 1, the SSM algorithm and SVD algorithm completely failed to work, which unable to estimate coherent signal spectrum peak, and the Toeplitz algorithm can barely able to estimate the signal peak, but have a slight deviation. However, the proposed algorithm can accurately estimate the spectrum peaks of three coherent signals which have no significant difference between to that of large number snapshot.

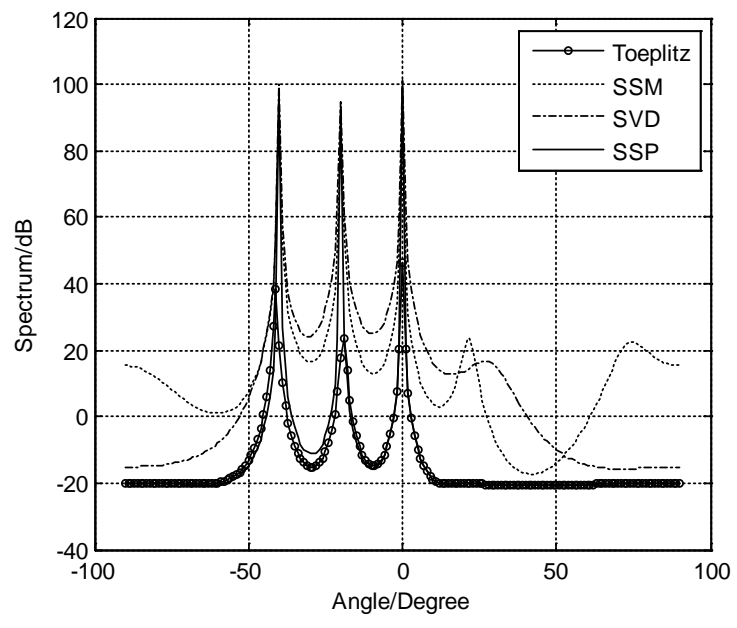

Figure .1 Algorithm performance simulation of snapshot number 1000

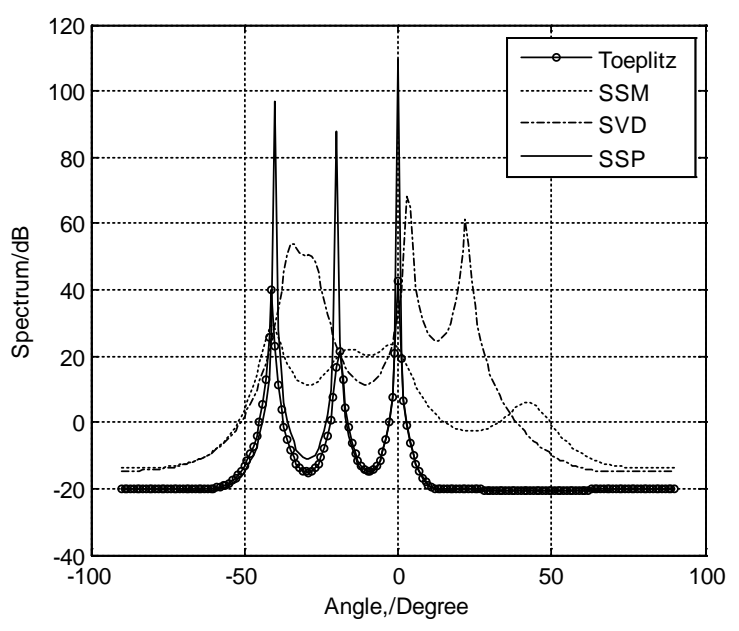

Figure .2 Algorithm performance simulation of snapshot number 1

Simulation 2: the effect of incident angle on the algorithm.

In this simulation, we assumed that three target signals of equal power with angles $\theta_{1}=-40^{\circ}, \theta_{2}=-35^{\circ}, \theta_{3}=0^{\circ}$ and $S N R=20 \mathrm{~dB}$, using music algorithm for spectral peak searching, the searching precision is $1^{\circ}$. When the snapshot number is 1000 , the algorithm in this paper, the simulation results of the algorithm of Toeplitz, SSM, SVD and SSP are shown in Figure.3. It can be seen from Figure. 3 that due to the loss of the array aperture, it is difficult for the other three algorithms to obtain the orthogonal subspace to the signal and the noise subspace, and it is difficult to estimate two target signals with similar angle of incidence (a difference of 5 degrees).The proposed algorithm can accurately estimate the two adjacent signals due to preserve the aperture of the array. When the snapshot number is 1 with other simulation conditions not changed, the simulation results is shown in figure 4. Obviously, when the sample data is small, the algorithm can still estimate the two adjacent signals, which is not affected by the number of snapshots.

Simulation 3: The variation of proposed algorithm estimation error with signal to noise ratio

To avoid the influence of the resolution of the algorithm, we assume that, three target signals of equal power with angles $\theta_{1}=-40^{\circ}, \theta_{2}=-20^{\circ}, \theta_{3}=0^{\circ}$, for single snapshot data, $S N R$ changes from $-10 \mathrm{~dB}$ to $10 \mathrm{~dB}$ by step $2 \mathrm{~dB}$, using music algorithm for 100 times Monte Carlo simulation, The mean square error (RMSE) of the algorithm varies as shown in Figure.5. RMSE is defined as follows:

$$
R M S E=\left[\frac{1}{N \times P} \sum_{p=1}^{P} \sum_{i=1}^{N}\left(\hat{\theta}_{p}-\theta_{i}\right)^{2}\right]^{\frac{1}{2}}
$$

Among them, $N$ is the number of incident signal, $P$ is the number of independent experiments, $\theta_{i}$ is the standard value of the incident signal, $\hat{\theta}_{p}$ is the DOA estimation of target single. As can be seen from Figure 5, with the increase of $S N R$,the algorithm of estimation error decreased obviously. Compared with the other three algorithms, the proposed algorithm has lower RMSE than that of the 
other three algorithms in the low SNR scenarios. With the improvement of signal to noise ratio, the estimation errors of all algorithms tend to be consistent, and there is no significant difference.

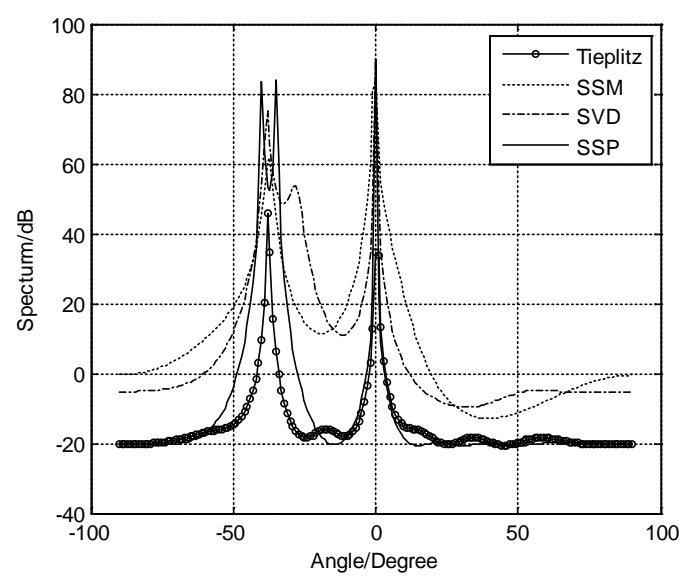

Figure .3 Algorithm Resolution of snapshot number 1000

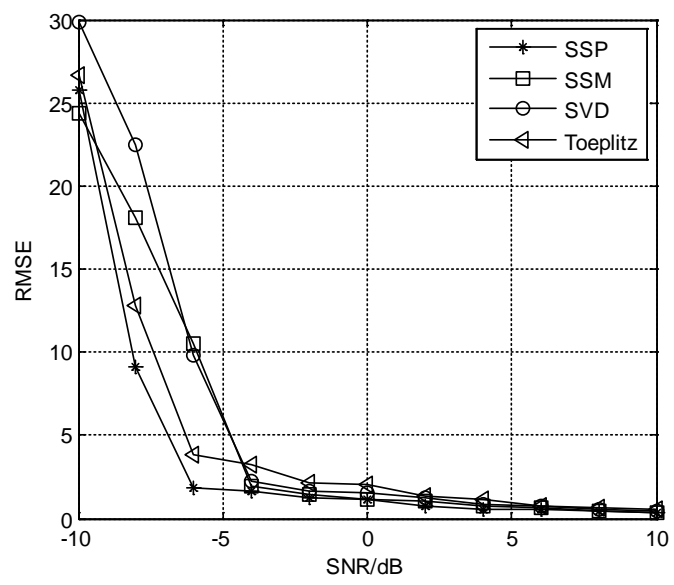

Figure .4 Algorithm estimation error simulation with SNR

\section{Conclusions}

In this paper, we have proposed a new single snapshot of coherent signal DOA estimation algorithm based on the analysis of the existing the limitations of decorrelation algorithms. very efficient DOA estimation method for weak coherent signals with strong jamming environment. Without assistance of the DOA information of the strong jamming signals ,firstly, via the modified Toeplitz matrix, the decorrelation of the coherent weak signals is carried out in this algorithm. Then, the feature vector of the strong jamming is removed from the signal subspace. Finally, the DOA of the weak signal is estimated with the MUSIC algorithm. A number of simulations demonstrate that our proposed method provides a better DOA estimation performance than traditional method, especially for correlated signals. The equivalent covariance matrix is reconstructed by using the single snapshot data, without loss of the array aperture, the algorithm can achieves better performance. The proposed algorithm has low computational complexity, and the estimation accuracy is better than that of the existing algorithms in small sample size. Computer simulation results verify the effectiveness of the proposed algorithm, which is suitable for the DOA estimation problem in high mobility scenarios.

\section{References}

[1] ZengHao, Zhu Zihua, Li Huijun and other [J]. uniform circular array of broadband signal DOA estimation algorithm [J]. circuits and systems, 2013,1 (18): 432-436.

[2] Shan T J, Wax M, Kailath T. On spatial smoothing for estimation of coherent signals[J].IEEE Trans on Acoustics, Speech and Signal Processing,1985,33(4):806-811.

[3] Cadzow J A, Kim Y S, Shiue D C. General direction-of-arrival estimation: A signal subspace approach[J].IEEE Trans on Aerospace and Electronic System,1989,25(1):31-47.

[4] CHEN Y M, Lee J H, Yeh C C, etal. Bearing estimation without calibration for randomly perturbed arrays[J].IEEE Trans on Signal Processing,1991,39(1):194-197.

[5] Cadzow J A. A high resolution direction-of-arrival algorithm for narrow-band coherent and incoherent sources[J].IEEE Trans on Acoustics, Speech and Signal Processing,1988,36(7):965-979.

[6] Thakre A, Haardt M, Giridhar K. Single snapshot spatial smoothing with improved effective array aperture [J]. Signal Processing,2012,92(10):2566-2570.

[7] WEI Yin-sheng, TONG Peng, GUO Xiao-jiang. Signal snapshot super resolution algorithm for 
HFSWR based on noise eigenvector reconstruction[J].Systems Engineering and Electronics,2013,35(3):493-498.

[8] WANG Ling, LI Guo-lin,LI Jing, etal.A novel single snapshot two-dimensional ESPRIT algorithm[J].Transactions of Beijing Institute of Technology,2013,33(1):99-104. 